283

SUPPORTING EVIDENCE TO DIFFERENTIATE PREECLAMPSIA FROM NEPHRITIS IN LUPUS PREGNANCY: AN ELECTRONIC HEALTH RECORDS REVIEW

Titilola Falasinnu*, Julia F Simard. Stanford School of Medicine

\subsection{6/lupus-2019-Ism.283}

Background Pregnancy in women with SLE is associated with a greater risk of both maternal and neonatal complications. Preeclampsia in SLE is particularly tricky because its manifestations and symptoms are nearly indistinguishable from lupus nephritis, a serious SLE manifestation. Renal biopsy is indicated in SLE if active nephritis is suspected, however this is not without risks and many patients and providers prefer to avoid such invasive procedures in later pregnancy. Much of the work on preeclampsia in lupus has relied on clinical documentation and coding, which may be subject to substantial misclassification. We aimed to describe the extent to which the clinical features (purported to support distinguishing between flare/nephritis and preeclampsia) are recorded in electronic health records (EHR).

Methods We identified a cohort of pregnant women with SLE from a tertiary medical teaching hospital using both ICD coded visits and medical record review (excluding drug-induced lupus M32.0) in the EHR. From these pregnancies, we identified 23 women with preeclampsia noted by their treating physician on medical record review. We evaluated documentation and/or measurement of important diagnostic features such as complement, antibodies to double stranded DNA (anti-dsDNA), differential blood counts, the presence of cellular and acellular blood casts, urinalysis, liver function tests, uric acid levels, and the presence of schistocytes. We recorded the percentage of time that these measures were noted in the EHR. We repeated this restricting to a period with more complete EHR in a sensitivity analysis.

Results Missingness was highest for blood smears to determine the presence of schistocytes (91\%). We found that complement levels were not recorded for $51 \%$ of patients, while anti-dsDNA was missing in $43 \%$ of patients. Differential blood counts were recorded for all patients. We could not find evidence for microscopic urinalysis to rule out casts or hematuria in $\sim 9 \%$ of cases. Uric acid levels were not measured in $13 \%$ of patients and liver function tests were missing in 39\%. We found attenuation of missingness in contemporary cases, but only to a minimal effect.

Conclusions We found that the diagnosis of preeclampsia in this setting was not supported by strong recorded evidence of clinical features upon extensive chart reviews (above and beyond proteinuria and hypertension, two factors that may also be present in nephritis). All of these women diagnosed with preeclampsia also delivered preterm, however, we did not find extensive documentation attempting to distinguish preeclampsia from potential nephritis flare, which would necessitate a different treatment.

Funding Source(s): NIAMS

\section{T CELLS PRODUCE INTERFERON ALPHA IN A MODEL OF LUPUS-LIKE DISEASE}

${ }^{1}$ Sean Simpson*, ${ }^{2}$ Stephen Rego, ${ }^{2}$ Scott Harvey, ${ }^{2}$ Jason Grayson, ${ }^{2}$ Fred Perrino. ${ }^{1}$ Wake Forest School of Medicine; ${ }^{2}$ Wake Forest University School of Medicine

10.1136/lupus-2019-Ism.284

Abstract 284 Figure 1 TREX1 D18N T cells spontaneously generate IFN-a. (A) Kaplan-Meir survival curve demonstrating the absence of autoimmune mortality in TREX1 D18N IFNAR KO animals. (B) qRT-PCR measurement of IFN- gene expression in uninfected WT splenocytes, LCMV +splenocytes, purified LCMV + non innate immune cells, and purified LCMV +innate immune cells (data represents cells from three virallychallenged mice 48 hours after infection). (C) IFN- gene expression in splenocytes from unchallenged WT and D18N mice (26-30 mice per genotype) (D) IFN- gene expression in whole splenocytes and purified non-innate and innate immune cells from both WT and D18N mice (3 separations, 6-8 mice per genotype) (E) qRT-PCR measurement of IFN- expression or ISG expression (F) in whole splenocytes or purified T and B cells from WT and D18N mice (3 separations, 3 mice of each genotype). (G) IFN- expression in sorted naïve or differentiated CD44high CD4 or CD8 T cells from both WT and D18N mice (3 independent sorts, 3 animals of each genotype). All mice were mixed sex and 8-12 weeks of age. Error bars represent SEM. ${ }^{*} p<0.05,{ }^{* *} p<0.01,{ }^{* * *} p<0.001{ }^{* * * *} p<0.0001$ by Log-rank test $(A)$, unpaired student's t-test (C, WT vs. D18N comparisons in D-G), or one-way ANOVA (B, D18N splenocytes vs. other D18N populations in D-G). 